\title{
Maximum likelihood trajectories from single molecule fluorescence resonance energy transfer experiments
}

\author{
Gunnar F. Schröder and Helmut Grubmüller ${ }^{a)}$ \\ Theoretical Molecular Biophysics Group, Max-Planck-Institute for Biophysical Chemistry, Am Fassberg 11, \\ 37077 Göttingen, Germany
}

(Received 4 June 2003; accepted 14 August 2003)

\begin{abstract}
Single molecule fluorescence resonance energy transfer (FRET) experiments are a powerful and versatile tool for studying conformational motions of single biomolecules. However, the small number of recorded photons typically limits the achieved time resolution. We develop a maximum likelihood theory that uses the full information of the recorded photon arrival times to reconstruct nanometer distance trajectories. In contrast to the conventional, intensity-based approach, our maximum likelihood approach does not suffer from biased a priori distance distributions. Furthermore, by providing probability distributions for the distance, the theory also yields rigorous error bounds. Applied to a burst of 230 photons obtained from a FRET dye pair site-specifically linked to the neural fusion protein syntaxin-1a, the theory enables one to distinguish time-resolved details of millisecond fluctuations from shot noise. From cross validation, an effective diffusion coefficient is also determined from the FRET data. (C) 2003 American Institute of Physics.
\end{abstract}

[DOI: $10.1063 / 1.1616511]$

\section{INTRODUCTION}

Fluorescence resonance energy transfer (FRET) measurements allow one to determine the distance between two dyes at a nanometer scale. ${ }^{1-3}$ In a typical set-up (Fig. 1), information on the structure of a biomolecule such as DNA or a protein is obtained from a pair of FRET dyes, a donor and an acceptor, which are covalently attached at defined positions to the biomolecule. After excitation of the donor, and depending on the distance and relative orientation between the two dyes, energy is transferred to the acceptor by the Förster mechanism. ${ }^{1}$ Thus, by measuring donor and acceptor fluorescence intensities, $I_{D}$ and $I_{A}$, the distance $r$ between the two dyes is obtained, usually via

$$
\frac{I_{A}}{I_{A}+I_{D}}=\frac{1}{1+\left(\frac{r}{r_{0}}\right)^{6}},
$$

where $r_{0}$ is the dye-specific effective Förster radius, which also includes (averaged) dye orientation effects. ${ }^{2}$ This approach is valid if the relative dye rotations are faster than the lifetime of the excited state of the donor, which is usually the case.

Recently, time-resolved FRET experiments have matured to a level that allows one to record arrival times of individual photons from single molecules. ${ }^{4-11}$ From the arrival times, fluorescence intensity variations, $I_{D}(t)$ and $I_{A}(t)$, are obtained, ${ }^{10,12,13}$ which, using Eq. (1), allow one to track distance changes $r(t)$ between the two dyes, and hence to monitor conformational motions of the studied biomolecule. $^{12,13}$

\footnotetext{
a)Phone: ++49-551-201-1763; Fax: ++49-551-201-1089; Electronic mail: hgrubmu@gwdg.de
}

In the conventional analysis, the required FRET intensities are computed from photon counts in time windows 8,10 (cf. also Ref. 14). For a typical window size of $1 \mathrm{~ms}$, however, the small number of only $10-50$ photons per window ${ }^{10}$ implies considerable statistical uncertainty ("shot noise" 15 ) and thus limits the time resolution for $r(t)$. Furthermore, the choice of the window size is somewhat arbitrary and only guided by the requirement to trade off shot noise and time resolution. Finally, the traditional method saliently assumes a uniform a priori probability for the FRET intensities (rather than for the distances). Therefore, and contrary to what one might intuitively assume at first sight, the traditional method cannot be considered a model-free approach. Rather, because the distance $r$ depends nonlinearly on the intensities, Eq. (1), the assumed uniform intensity distribution transforms into a nonuniform distance distribution,

$$
p(r)=\frac{\left(\frac{r}{r_{0}}\right)^{5}}{\left[1+\left(\frac{r}{r_{0}}\right)^{6}\right]^{2}} .
$$

This distribution is centered at the Förster radius and has a half width of about $\frac{1}{3} r_{0}$, implying preferred distances near $r_{0}$; it describes the unjustified bias introduced by the conventional analysis.

In many cases where only limited or noisy data are available, the maximum-likelihood approach has been successfully applied. ${ }^{16-22}$ In this article, we develop a maximumlikelihood theory to reconstruct $r(t)$ from the photons recorded in single molecule FRET measurements. In particular, we aim at calculating the time-dependent probability distribution $P\left(r, t \mid\left\{t_{i}^{D}, t_{i}^{A}\right\}\right)$ for the distance $r$ during a measurement of length $\Delta T$, given that $n_{D}$ photons from the donor dye have been recorded at times $t_{i}^{D}, i=1, \ldots, n_{D}$, and $n_{A}$ 


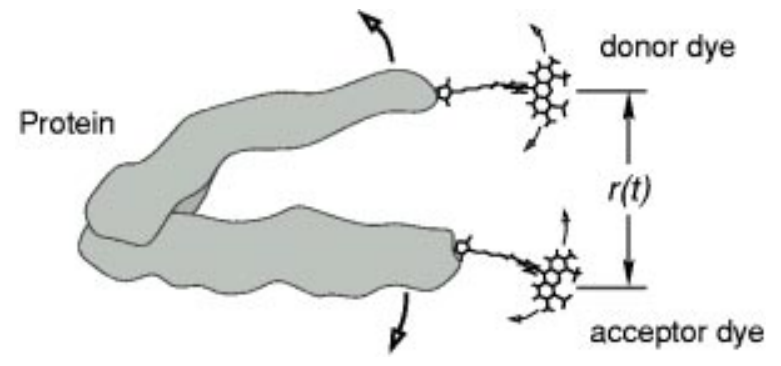

FIG. 1. Typical single molecule FRET experiment. A donor and an acceptor dye molecule are attached to a protein that exhibits conformational dynamics. By probing the interdye distance trajectory $r(t)$, measurement of the FRET efficiency provides time-resolved information on the dynamics of the studied protein (arrows).

acceptor photons at times $t_{i}^{A}, i=1, \ldots, n_{A}$. Finally, we will extract an effective diffusion coefficient for the biomolecular motion from the FRET data.

\section{THEORY}

To that aim, in a first step we consider a statistical ensemble of distance trajectories, $\{r(t)\}$, and compute for each full trajectory the conditional probability $P\left[r(t) \mid\left\{t_{i}^{D}, t_{i}^{A}\right\}\right]$ that $r(t)$ is realized for the given photon registration times. Assuming Bayesian statistics, this probability is given by the a priori probability $P[r(t)]$ for each trajectory and the conditional probability that the $n_{A}+n_{D}$ photons are observed at the measured time instances for given trajectory,

$$
P\left[r(t) \mid\left\{t_{i}^{D}, t_{i}^{A}\right\}\right] \propto P[r(t)] P\left[\left\{t_{i}^{D}, t_{i}^{A}\right\} \mid r(t)\right] .
$$

To evaluate these two distributions, the time interval $\Delta T$ is discretized into $N$ bins $\left[\tau_{j-1}, \tau_{j}\right], j=1, \ldots, N$, and subsequently $N \rightarrow \infty$ is considered. The time discretization $\tau:=\tau_{j}$ $-\tau_{j-1}=\Delta T / N$ is always chosen fine enough such that not more than one photon per interval $\left[\tau_{j-1}, \tau_{j}\right]$ is recorded.

For a discretized trajectory $r_{1}, \ldots, r_{N}$, where $r_{j}$ is the distance at time $\frac{1}{2}\left(\tau_{j-1}+\tau_{j}\right)$, the conditional probability to observe the recorded photon pattern $E_{1}, \ldots, E_{N}$ is

$$
P\left[E_{1}, \ldots, E_{N} \mid r_{1}, \ldots, r_{N}\right]=\tau^{n_{A}+n_{D}} \prod_{j=1}^{N} f_{j},
$$

where the probabilities $f_{j}$ are chosen according to which of the three possible events $E_{j}$ [donor-photon is recorded $(D)$, acceptor-photon is recorded $(A)$, or no photon is recorded (0)] occurs during $\left[\tau_{j-1}, \tau_{j}\right]$,

$$
f_{j}= \begin{cases}I_{D}\left(r_{j}\right)\left[1-\tau I_{A}\left(r_{j}\right)\right] & \text { for } D, \\ I_{A}\left(r_{j}\right)\left[1-\tau I_{D}\left(r_{j}\right)\right] & \text { for } A, \\ {\left[1-\tau I_{D}\left(r_{j}\right)\right]\left[1-\tau I_{A}\left(r_{j}\right)\right]} & \text { for } 0 .\end{cases}
$$

Here, $I_{A}\left(r_{j}\right)$ and $I_{D}\left(r_{j}\right)$ are specified from Eq. (1), and the required (average) total intensity $I_{0}=I_{A}(t)+I_{D}(t)=\left(n_{A}\right.$ $\left.+n_{D}\right) / \Delta T$ is estimated from the recorded number of photons. Note that for the $n_{D}+n_{A}$ events $D$ and $A$, the $f_{j}$ denote probability densities, which have to be scaled by $\tau$ to obtain the desired probabilities, hence the prefactor in Eq. (4).

For the a priori probability $P[r(t)]$ $\propto \lim _{N \rightarrow \infty} P\left[r_{1}, \ldots, r_{N}\right]$, we assume that $r(t)$ results from a one-dimensional diffusion process with effective diffusion coefficient $D$. This is realistic, e.g., for the overdamped millisecond opening and closure domain motions of the solvated macromolecule at hand. ${ }^{10}$ The discretized version is a random walk with transition probabilities

$$
g_{j+1 \mid j} \propto \frac{1}{\sqrt{4 \pi D \tau}} \exp \left[-\frac{\left(r_{j+1}-r_{j}\right)^{2}}{4 D \tau}\right] .
$$

Note that this implies that all possible distances are assigned equal a priori probabilities, which is reasonable if the energy landscape that governs the distance distribution is unknown. If there is additional information on the energy landscape, this can be incorporated into $g_{j+1 \mid j}$ in a Smoluchowsky-type generalization. Note also that two or three dimensional diffusion of the dyes can be described in a similar manner by an appropriate effective energy landscape that accounts for the projection of the higher-dimensional diffusion onto the onedimensional distance coordinate $r(t)$. Thus, $P\left[r_{1}, \ldots, r_{N}\right]$ $=\Pi_{j=2}^{N} g_{j \mid j-1}$, and Eq. (3) reads

$$
P\left[r_{1}, \ldots, r_{N} \mid\left\{t_{i}^{D}, t_{i}^{A}\right\}\right] \propto f_{1} \prod_{j=2}^{N} g_{j \mid j-1} f_{j} .
$$

In a second step the probability distribution for the distance $r_{k}$ at times $\left(\tau_{k-1}+\tau_{k}\right) / 2$ is calculated by integration over all other distances,

$$
\begin{aligned}
P\left(r_{k} \mid\left\{t_{i}^{D}, t_{i}^{A}\right\}\right) \propto & \int \cdots \int d r_{1} \cdots d r_{k-1} d r_{k+1} \cdots \\
& d r_{N} P\left[r_{1}, \ldots, r_{N} \mid\left\{t_{i}^{D}, t_{i}^{A}\right\}\right] .
\end{aligned}
$$

Using Eq. (7) and rearranging integrals, one obtains

$$
P\left(r_{k} \mid\left\{t_{i}^{D}, t_{i}^{A}\right\}\right) \propto L_{k} f_{k} R_{k}
$$

with

$$
\begin{aligned}
& L_{k}=\int d r_{k-1} g_{k \mid k-1} f_{k-1} \int d r_{k-2} \cdots \int d r_{1} g_{2 \mid 1} f_{1}, \\
& R_{k}=\int d r_{k+1} g_{k+1 \mid k} f_{k+1} \int d r_{k+2} \cdots \int d r_{N} g_{N \mid N-1} f_{N} .
\end{aligned}
$$

The above two equations obey the recursion relations

$$
\begin{aligned}
& L_{k}=\int d r_{k-1} g_{k \mid k-1} f_{k-1} L_{k-1}, \\
& R_{k}=\int d r_{k+1} g_{k+1 \mid k} f_{k+1} R_{k+1},
\end{aligned}
$$

which, in the continuum limit (i.e., $\tau \rightarrow 0, \tau_{j} \rightarrow t$, and $r_{k}$ $\rightarrow r$ ), transform into forward and backward Schrödinger-type equations that resemble generalized diffusion equations for $L_{k} \rightarrow L(r, t)$ and $R_{k} \rightarrow R(r, t)$,

$$
\begin{aligned}
\partial_{t} L(r, t)= & \lim _{\tau \rightarrow 0}\left\{\partial_{r}^{2}\left[\left(1+\tau F_{\tau}(r, t)\right) L(r, t)\right]\right. \\
& +\left[F_{\tau}(r, t)+\tau \partial_{\tau} F_{\tau}(r, t)\right] L(r, t), \\
\partial_{t} R(r, t)= & -\lim _{\tau \rightarrow 0}\left\{\partial_{r}^{2}\left[\left(1+\tau F_{\tau}(r, t)\right) R(r, t)\right]\right. \\
& \left.+\left[F_{\tau}(r, t)+\tau \partial_{\tau} F_{\tau}(r, t)\right] R(r, t)\right\}
\end{aligned}
$$


where, to ensure convergence, $f_{k}$ has been written in the form $f_{k}=1+\tau F_{\tau}(r, t)$. For the derivation of Eqs. (12), the recursion relations Eqs. (11) have been expanded in $\tau$ up to first order, using $\partial_{\tau} g_{k \mid k-1}=D \partial_{r_{k-1}}^{2} g_{k \mid k-1}=D \partial_{r_{k}}^{2} g_{k \mid k-1}$, and partial integration in $r$, noting that $L(r, t)$ and $R(r, t)$ as well as their derivatives with respect to $r$ vanish for $r \rightarrow \pm \infty$.

Solving Eqs. (12) yields, after normalization, the desired probability distribution to find the distance $r$ at time $t$,

$$
P\left(r, t \mid\left\{t_{i}^{D}, t_{i}^{A}\right\}\right) \propto L(r, t)\left[1+\tau F_{\tau}(r, t)\right] R(r, t) .
$$

By combining the three definitions for $f_{j}$, Eq. (5), into one expression using a Gaussian limit representation for the $\delta$-function, $\delta\left(t-t^{\prime}\right)=\lim _{\tau \rightarrow 0} h_{\tau}\left(t-t^{\prime}\right)$, with

$$
h_{\tau}\left(t-t^{\prime}\right)=\frac{1}{\sqrt{2 \pi} \tau} \exp \left[-\frac{\left(t-t^{\prime}\right)^{2}}{2 \tau^{2}}\right] \text {, }
$$

and neglecting higher orders of $\tau$, one obtains

$$
\begin{aligned}
F_{\tau}(r, t)= & {\left[I_{D}(r)-1\right] \sum_{j=1}^{n_{D}} h_{\tau}\left(t-t_{j}^{D}\right) } \\
& +\left[I_{A}(r)-1\right] \sum_{j=1}^{n_{A}} h_{\tau}\left(t-t_{j}^{A}\right)-I_{0} .
\end{aligned}
$$

With this expression, Eqs. (12) reads

$$
\begin{aligned}
\partial_{t} L(r, t) & \lim _{\tau \rightarrow 0}\left\{\int d r ^ { \prime } g ( r - r ^ { \prime } , \tau ) \partial _ { r ^ { \prime } } ^ { 2 } \left[L\left(r^{\prime}, t\right)\right.\right. \\
& \times\left(1+\tau\left[I_{D}\left(r^{\prime}\right)-1\right] \sum_{j=1}^{n_{D}} h_{\tau}\left(t-t_{j}^{D}\right)+\tau\left[I_{A}\left(r^{\prime}\right)-1\right]\right. \\
& \left.\left.\times \sum_{j=1}^{n_{A}} h_{\tau}\left(t-t_{j}^{A}\right)\right)\right]+\int d r^{\prime} g\left(r-r^{\prime}, \tau\right) L\left(r^{\prime}, t\right) \\
& \times\left[\frac{I_{D}\left(r^{\prime}\right)-1}{\tau^{2}} \sum_{j=1}^{n_{D}}\left(t-t_{j}^{D}\right)^{2} h_{\tau}\left(t-t_{j}^{D}\right)+\frac{I_{A}\left(r^{\prime}\right)-1}{\tau^{2}}\right. \\
& \left.\left.\times \sum_{j=1}^{n_{A}}\left(t-t_{j}^{A}\right)^{2} h_{\tau}\left(t-t_{j}^{A}\right)-I_{0}\right]\right\} .
\end{aligned}
$$

A similar expression is obtained for $R(r, t)$. For times $t$, for which no photon arrives, Eq. (16) simplifies to

$$
\begin{aligned}
& \partial_{t} L(r, t)=D \partial_{r}^{2} L(r, t)-I_{0} L(r, t), \\
& \partial_{t} R(r, t)=-D \partial_{r}^{2} R(r, t)+I_{0} R(r, t),
\end{aligned}
$$

with solutions that propagate in time according to

$$
\begin{aligned}
& L(r, t)=e^{-I_{0}\left(t-t^{\prime}\right)} \int d r^{\prime} L\left(r^{\prime}, t^{\prime}\right) \exp \left[-\frac{\left(r-r^{\prime}\right)^{2}}{4 D\left(t-t^{\prime}\right)}\right], \\
& R(r, t)=e^{I_{0}\left(t^{\prime}-t\right)} \int d r^{\prime} R\left(r^{\prime}, t^{\prime}\right) \exp \left[-\frac{\left(r-r^{\prime}\right)^{2}}{4 D\left(t^{\prime}-t\right)}\right]
\end{aligned}
$$

for $t>t^{\prime}$ and $t<t^{\prime}$, respectively. To also include the photon arrival times $t_{j}$, note that

$$
\begin{aligned}
\lim _{\tau \rightarrow 0}\left(t-t_{j}\right)^{2} h_{\tau}\left(t-t_{j}\right) / \tau^{2} \\
\quad=\lim _{\tau \rightarrow 0} h_{\tau}\left(t-t_{j}\right)+\lim _{\tau \rightarrow 0} \tau^{2} \partial_{t}^{2} h_{\tau}\left(t-t_{j}\right) \\
\quad=\delta\left(t-t_{j}\right),
\end{aligned}
$$

where the second term is $\propto \partial_{t}^{2} \delta\left(t-t_{j}\right)$ and is dropped, because $\int_{-\epsilon}^{\epsilon} \delta^{\prime \prime}(x) d x=0$. This gives rise to additive singularities in Eqs. (17) of the form $L(r, t)\left[\left(I_{D}(r)-1\right)\right] \delta\left(t-t_{j}\right)$, due to which $L(r, t)$ and $R(r, t)$ exhibit discontinuities at all $t_{j}$,

$$
\begin{aligned}
& \lim _{t \rightarrow\left(t_{j}^{D}\right)^{+}} L(r, t)=I_{D}(r) \lim _{t \rightarrow\left(t_{j}^{D}\right)^{-}} L(r, t), \\
& \lim _{t \rightarrow\left(t_{j}^{A}\right)^{+}} L(r, t)=I_{A}(r) \lim _{t \rightarrow\left(t_{j}^{A}\right)^{-}} L(r, t), \\
& \lim _{t \rightarrow\left(t_{j}^{D}\right)^{-}} R(r, t)=I_{D}(r) \lim _{t \rightarrow\left(t_{j}^{D}\right)^{+}} R(r, t), \\
& \lim _{t \rightarrow\left(t_{j}^{A}\right)^{-}} R(r, t)=I_{A}(r) \lim _{t \rightarrow\left(t_{j}^{A}\right)^{+}} R(r, t) .
\end{aligned}
$$

Equations (18) and (20) are the main result of this article. Starting with the boundary condition $L(r, 0)=1$, Eqs. (18) and (20), when alternatingly applied, propagate $L(r, t)$ in time from one photon arrival to the next. Similarly, starting from $R(r, \Delta T)=1, R(r, t)$ is propagated in reverse time direction, which, by using Eq. (13), yields $P\left(r, t \mid\left\{t_{i}^{D}, t_{i}^{A}\right\}\right)$ for all times $t$. Note that, from Eqs. (20), the discontinuities in $L(r, t)$ and $R(r, t)$ cancel in Eq. (13), such that $P\left(r, t \mid\left\{t_{i}^{D}, t_{i}^{A}\right\}\right)$ is nondifferentiable, but continuous also at $t$ $=t_{j}$.

\section{RESULTS AND DISCUSSION}

As an example, Figs. 2(b)-2(d) show the application of our theory to the 230 photon arrival times (wedges) from a $10 \mathrm{~ms}$ single molecule photon burst recorded in a FRET measurement, for which donor and acceptor dyes have been covalently linked to the flexible domains of the neuronal fusion protein syntaxin-1a, ${ }^{10}$ as sketched in Fig. 1. Three different diffusion coefficients $D$ have been chosen. Each of the three plots shows, gray-shaded, the time dependent distance distribution $P\left(r, t \mid\left\{t_{i}^{D}, t_{i}^{A}\right\}\right)$, together with the average distance (bold) and $1 \sigma$ intervals (dashed). As expected from Eq. (1), larger distances are obtained for higher donor and lower acceptor photon intensities. For comparison, Fig. 2(a) shows the traditional method, which directly uses Eq. (1) with intensities and error bars evaluated in successive time bins, ${ }^{23}$ here of $0.5 \mathrm{~ms}$ width.

Apparently, the choice of $D$ is critical. For small values, the distance can change only slowly. Therefore, it does not fully reflect the significant intensity fluctuations encoded in the recorded photon arrival times, and rather yields smooth trajectories with small amplitude. For very small values (below $0.01 \times 10^{-14} \mathrm{~m}^{2} / \mathrm{s}$ ), the distance distribution becomes time independent and approaches the distance given by the average intensities (data not shown). Increasing $D$ entails fluctuations of correspondingly increased frequencies. These fluctuations arise from both intensity fluctuations due to actual distance variations and (undesirable) probability fluctua- 


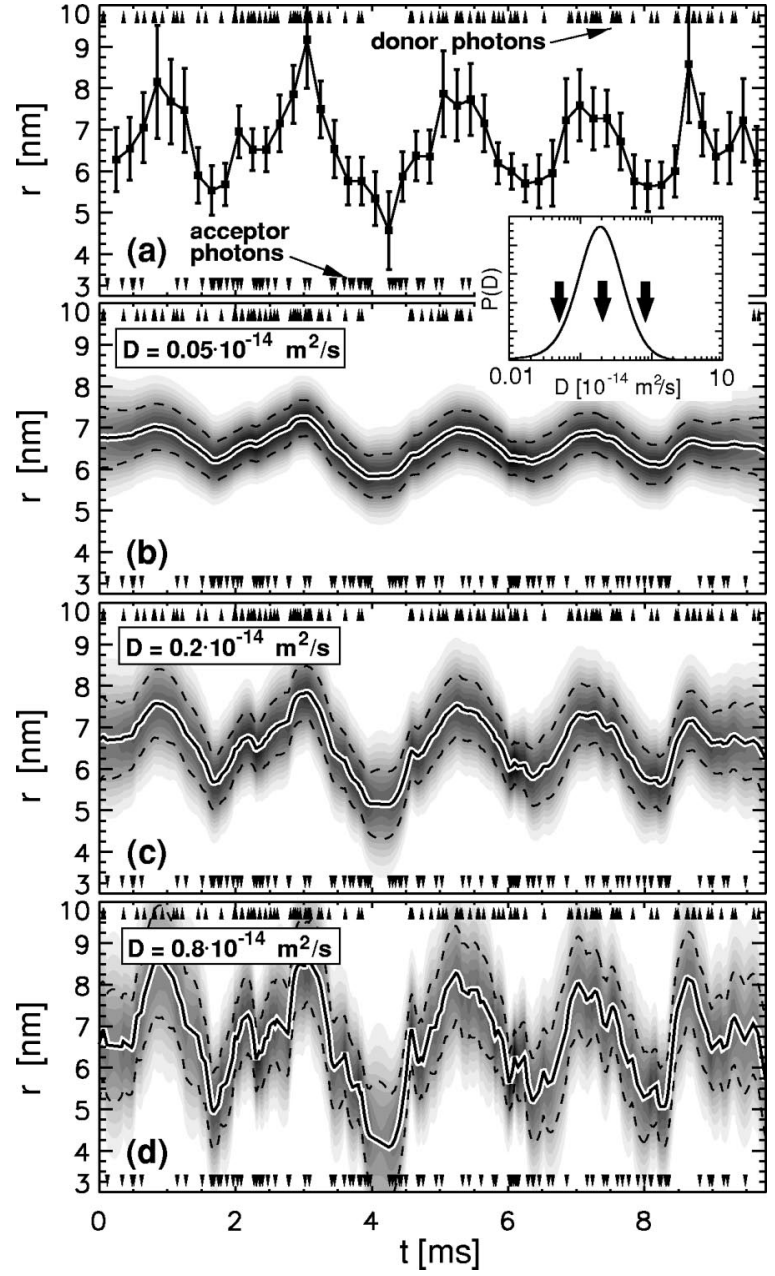

FIG. 2. (a) Intensity-based calculation of donor/acceptor distances $r(t)$ from a set of 230 photon arrival times (wedges) with $r_{0}=6.5 \mathrm{~nm}$ (Ref. 10) using Eq. (1); intensities are obtained from $0.5 \mathrm{~ms}$ bins. (b)-(d) Time dependent distance probability distributions $P\left(r, t \mid\left\{t_{i}^{D}, t_{i}^{A}\right\}\right)$ (gray-shaded) calculated from the same set for three different diffusion coefficients $D$. Also shown are average distance trajectories (bold) and $1 \sigma$ intervals (dashed). The inset shows the (normalized) likelihood $P(D)$ as a function of $D$; three arrows denote the three chosen values for $D$.

tions due to the broadening of $L(r, t)$ and $R(r, t)$ between subsequent photons. As can be seen from Eqs. (18), the latter become relevant for $4 D>I_{0} \sigma^{2}$, where $\sigma$ is the width of $P\left(r, t \mid\left\{t_{i}^{D}, t_{i}^{A}\right\}\right)$. The lower panel in Fig. 2 shows an example for which, due to the large $D$ chosen, the data are apparently overfitted. In between these two limiting cases, an optimal value for $D$ is expected to provide the best description of the data [Fig. 2(c)].

That optimal value was determined by calculating the agreement between the obtained time-dependent distance distribution and the measured photon arrival times as a function of the chosen $D$. Such type of cross-validation underlies, e.g., the free $R$ value used to assess the accuracy of macromolecular x-ray structures. ${ }^{24}$ In a similar spirit, one photon $k$ was excluded from the FRET data, and a new distance distribution,

$$
P_{k}\left(r_{k}\right) \equiv P_{k}\left(r_{k}, t_{k} \mid\left\{t_{i}^{D}, t_{i}^{A}, i \neq k\right\}\right)
$$

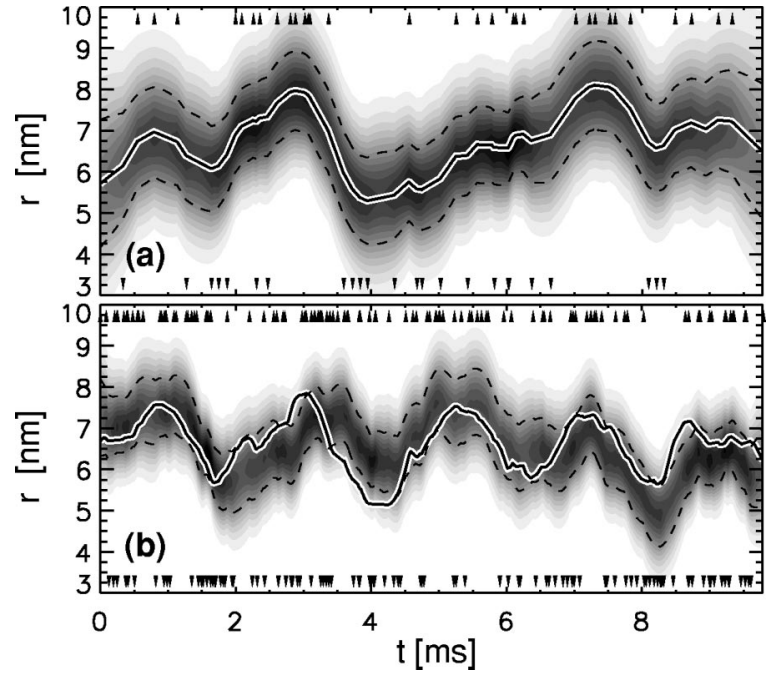

FIG. 3. (a) Distance distribution for a reduced set of 58 photons (wedges) and $D=0.2 \times 10^{-14} \mathrm{~m}^{2} / \mathrm{s}$; notation as in Fig. 2. (b) Recalculated distance distribution (gray-shaded) for a hypothetical set of 230 photons (wedges) that has been calculated from the original average trajectory in Fig. 2(c), also shown in bold here; $D=0.2 \times 10^{-14} \mathrm{~m}^{2} / \mathrm{s}$. The dashed lines denote the $1 \sigma$ interval for the recalculated distance distribution.

was obtained for the arrival time $t_{k}$ of the excluded photon. Using this distribution, the likelihood $P_{k}(D)$ for the actually observed photon $k$ was determined for varying $D$,

$$
P_{k}(D) \propto \int_{0}^{\infty} d r_{k} P_{k}\left(r_{k}\right) I_{D / A}\left(r_{k}\right)
$$

with $I_{D / A}$ chosen according to the type of the excluded photon. Assuming that for different photons $k$ chosen to be omitted, the obtained likelihoods $P_{k}(D)$ are statistically independent, one obtains from the maximum of the (normalized) joint likelihoods $P(D) \propto \Pi_{k} P_{k}(D)$ (inset of Fig. 2) a diffusion coefficient $D=0.2 \times 10^{-14} \mathrm{~m}^{2} / \mathrm{s}$ that describes the measured photon arrival times best. In the figure, no scale for $P(D)$ is given to avoid its erroneous interpretation as the (absolute) probability that $D$ is the correct diffusion constant.

Clearly, the fewer photons are available, the less information on $r(t)$ can be obtained. As an extreme case, Fig. 3(a) shows the result of our analysis with only every fourth photon from the original data used. As expected, the distance distribution becomes broader, and only some of the features seen in Fig. 2 remain. Yet, despite the very small number of photons used (58), our analysis still reveals a statistically significant distance fluctuation at the $1 \sigma$ level. This finding suggests that a correspondingly improved time resolution can be achieved by our method.

To check whether the width of the calculated distance distribution correctly describes the actual statistical uncertainty, we have finally used the average trajectory calculated from the original data [thick line in Fig. 2(c)] to create a new (hypothetical) set of 230 random photon arrival times obeying Eq. (1). Thus, for these data, the underlying trajectory is known. From that set, a new distance distribution was recalculated and compared with the correct trajectory [Fig. 3(b)]. 
As can be seen, most of the correct trajectory (bold) stays within the $1 \sigma$-range of the recalculated distance distribution, thus showing the reliability of our method.

We have developed a theory that enables reconstruction of nanometer distance trajectories from single molecule single photon FRET recordings. In contrast to the commonly used method of window averaging, the full single photon information is used, and rigorous error bounds are obtained. Furthermore, the method is expected to be robust with respect to variation of the excitation intensity $I_{0}$, e.g., due to diffusion of the particle through the laser focus. In addition, our approach allows to extract an effective diffusion constant from the FRET recordings and thus avoids the usual ad hoc choice of an averaging interval for the determination of intensities. Finally, the likelihood approach avoids the severe bias of usual distance determination due to the salient assumption of uniform a priori probabilities for the FRET intensities, which implies, via Eq. (1), preferred distances near $r_{0}$. Possible extensions of the method concern position- and dye-dependent detection efficiencies. Because low count rates are also often encountered for many other types of single molecule experiments, we expect our approach to be of wide applicability. A software package that implements this theory (FRETtrace) can be downloaded from the webpage of the authors.

\section{ACKNOWLEDGMENTS}

We thank C. Seidel for providing his FRET data, for valuable discussions, and for carefully reading the manuscript. This work was supported by the Volkswagen Foundation, Grant No. I/75 321.
${ }^{1}$ T. Förster, Ann. Phys. (Leipzig) 2, 55 (1948).

${ }^{2}$ B. W. van der Meer, G. Cooker, and S.-Y. Chen, Resonance Energy Transfer: Theory and Data (VCH, New York, 1994).

${ }^{3}$ S. H. Lin, W. Z. Xiao, and W. Dietz, Phys. Rev. E 47, 3698 (1993).

${ }^{4}$ S. Weiss, Science 283, 1676 (1999).

${ }^{5}$ S. Weiss, Nat. Struct. Biol. 7, 724 (2000).

${ }^{6}$ T. J. Ha, A. Y. Ting, J. Liang, W. B. Caldwell, A. A. Deniz, D. S. Chemla, P. G. Schultz, and S. Weiss, Proc. Natl. Acad. Sci. U.S.A. 96, 893 (1999).

${ }^{7}$ W. E. Moerner and Michel Orrit, Science 283, 1670 (1999).

${ }^{8}$ X. Zhuang, L. E. Bartley, H. P. Babcock, R. Russell, T. Ha, D. Herschlag, and S. Chu, Science 288, 2048 (2000).

${ }^{9}$ N. L. Goddard, G. Bonnet, O. Krichevsky, and A. Libchaber, Phys. Rev. Lett. 85, 2400 (2000).

${ }^{10}$ M. Margittai, J. Widengren, E. Schweinberger, et al., (unpublished).

${ }^{11}$ X. Zhuang, H. Kim, M. J. B. Pereira, H. P. Babcock, N. G. Walter, and S. Chu, Science 296, 1473 (2002).

${ }^{12}$ T. J. Ha, A. Y. Ting, J. Liang, A. A. Deniz, D. S. Chemla, P. G. Schultz, and S. Weiss, Chem. Phys. 247, 107 (1999).

${ }^{13}$ R. Kühnemuth and C. A. M. Seidel, Single Mol. 2, 251 (2001).

${ }^{14}$ E. Barkai, Y. J. Jung, and R. Silbey, Phys. Rev. Lett. 87, 207403 (2001).

${ }^{15}$ C. Eggeling, J. R. Fries, L. Brand, R. Gunther, and C. A. M. Seidel, Proc. Natl. Acad. Sci. U.S.A. 95, 1556 (1998).

${ }^{16}$ F. G. Ball, Y. Cai, J. B. Kadane, and A. O'Hagan, Proc. R. Soc. London, Ser. A 455, 2879 (1999).

${ }^{17}$ W. A. Carrington, R. M. Lynch, E. D. W. Moore, G. Isenberg, K. E. Fogarty, and F. S. Fredric, Science 268, 1483 (1995).

${ }^{18}$ T. Dudok de Wit and E. Floriani, Phys. Rev. E 58, 5115 (1998).

${ }^{19}$ T. J. Loredo and D. Q. Lamb, Phys. Rev. D 65, 063002 (2002).

${ }^{20}$ J. Enderlein, Appl. Opt. 34, 514 (1995).

${ }^{21}$ C. Zander, M. Sauer, K. H. Drexhage, D.-S. Ko, A. Schulz, J. Wolfrum, L. Brand, C. Eggeling, and C. A. M. Seidel, Appl. Phys. B: Lasers Opt. 63, 517 (1996).

${ }^{22}$ J. Enderlein, P. M. Goodwin, A. Van Orden, W. P. Ambrose, R. Erdmann, and R. A. Keller, Chem. Phys. Lett. 270, 464 (1997).

${ }^{23}$ P. J. Rothwell, S. Berger, O. Kensch, S. Felekyan, M. Antonik, B. M. Wöhrl, T. Restle, R. S. Goody, and C. A. M. Seidel, Proc. Natl. Acad. Sci. U.S.A. 100, 1655 (2003).

${ }^{24}$ A. Brünger, Nature (London) 355, 472 (1992). 